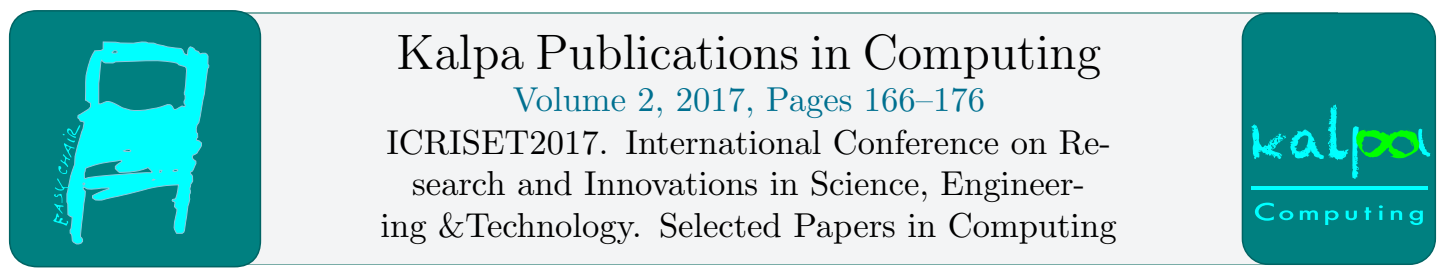

\title{
An Improved SimBet Routing Algorithm for Human Mobility based DTN
}

\author{
Mehul Shah ${ }^{1 *}$ Nikhil Godaliya ${ }^{2}$ and Avani Barad ${ }^{3}$ \\ ${ }^{1}$ BVM, V.V.Nagar, India \\ ${ }^{2}$ GCET, V.V.Nagar, India \\ ${ }^{2}$ Infosys, Bengaluru, India \\ mehul.shah@bvmengineering.ac.in, nikhilgondaliya@gcet.ac.in, \\ avanibarad05egmail.com
}

\begin{abstract}
Delay Tolerant Network (DTN) is useful for providing communication, where internet connection does not exist or in an Environment where long delay path and frequent partitions in transmission is present. A critical challenge for DTN is to determine the routing path through the network without ever having an end to end path, or finding which routers will be connected at any given instant of time. To find a routing path mobility of user needs attention, here we use human mobility model: Self Similar Least Action Walk (SLAW). In this paper, we extract pause time from past history of human mobility and utilize for routing in DTN. This concept will improve routing performance. In this paper, we propose the Multi-copy SimBet Routing with pause time factor. Finally, we conclude that the proposed routing method gives a message delivery ratio nearly equal to the Epidemic Routing, also reduce latency and overhead of the network.
\end{abstract}

\section{Introduction}

The DTN introduced in 2003[1], unlike TCP/IP based internet DTN has no end-to-end connection. DTN was originally developed for deep space communication, but now days it has drawn much attention due to its practical application in a communication challenge environment such as Terrestrial Mobile Networks, Exotic Media Networks, Military Ad-Hoc Networks and Sensor and Sensor/Actuator Networks [1]. Nowadays its practical implementation [2], [3] is also available. The unit of information exchange in a DTN is a bundle; the bundle layer is placed below the application layer, and hides the actual network or region specific communication layers [3].

\footnotetext{
* created the first stable version of this document
} 
DTN follows the store carry and forward [4] scenario, which occupies large buffer space, so to perform routing in an efficient way such that the performance of the network is not degraded, is a big challenge.

DTN has vital and challenging problem and that is to develop a forwarding mechanism in the communication challenge environment where nodes assumed to experience frequent, long-duration partitioning and may never have an end-to-end contemporaneous path [5]. The survey categorizes routing strategies of DTN using two properties; named as Flooding and Forwarding [6], [7]. In flooding property, multiple copies of message are spread over the network while in forwarding single copy of the message is transferred through carefully selected path using different routing scheme. Most of the DTN routing protocols belong to three categories: message-ferry-based, opportunitybased and prediction-based [8], but lately it considers social-characteristics like centrality, similarity, community and friendship for routing, as these characteristics are long term and less volatile than node mobility [8].

Humans carry wireless devices like laptop and cell phones, so the protocols should be tested under human mobility instead of random mobility models. This will decide the usefulness of a routing protocol.

As discussed above in this paper mobility of user is defined by human mobility model SLAW. SLAW traces have statistical resemblance with human walk. SLAW uses the characteristics like flight length, pause time, heterogeneously bounded mobility areas, Inter-Contact Times (ICTs), Fractal waypoints, Least-Action Trip Planning (LATP) [15]. These all discuss in detail in part B of section II. Part A of Section II discusses, work related to the existing routing algorithm. The proposed routing algorithm is discussed in Section III. Section IV contains brief about simulation setup and results of existing and proposed routing algorithms, and then we conclude work in Section V.

\section{Related Work}

Related work considers two categories, work based on existing routing protocols and work based on human mobility model.

\subsection{Routing Protocols}

This section considers description of existing routing protocols, which performance, we are comparing in Section IV.

\section{1) Epidemic}

The nature of Epidemic routing [9] is flooding-based, as nodes continuously replicates and forward messages to newly detected contacts that do not already know a copy of the message. The goal of Epidemic routing is to maximize efficiency by getting a higher message delivery ratio and lower latency, but epidemic give a higher overhead ratio because of the number of message copies flooded in the network. Epidemic routing follows the eventual delivery of messages to destinations. Epidemic routing has very less assumptions about network topology and connectivity of the whole network, for eventual delivery of message only periodic pair wise connectivity is required. In Epidemic, routing each host contains buffer for originated messages and carry messages for other hosts. The buffer size of each host is limited by the memory capacity of network and resource used by Epidemic routing. Maximum buffer size is set for each host to do message distribution. Epidemic follows FIFO [9] policy, as new messages arrive in system, older messages is deleted to clear memory for the new user host. 
Epidemic routing is designed to maximize message delivery rate and to minimize message delay while also minimizing the total resources consumed in message delivery by deleting older messages [9].

\section{2) Spray and Wait}

Spray and Wait routing developed to optimize the resource utilization. In Epidemic due to multiple copies of message, maximum resources are used while Spray and Wait routing algorithm limit replication of message by routing mechanism.

The Spray and Wait protocol is made up of two stages: the spray stage and the wait stage. When a new message is generated in the system, a number " $\mathrm{N}$ " is multiplied to that message copy to indicate the maximum permissible copies of that message in the network. In the spray stage, the source of the message is sprayed, or transmits, one copy to $\mathrm{N}$ different relays. When a relay receives the copy, algorithm enters in the wait stage, where the relay simply holds that particular message copy until the destination node is come in direct contact range [10]. Spray and Wait routing protocol occupies the buffer size until an encounter for message occurs, once encounter occurs, it clears the buffer space, but delay in the network will increase. In wait stage of the algorithm, it waits until an encounter for all messages occur, so overhead in the network is reduced as time passing.

\section{3) ProPhet}

The ProPhet is Probabilistic Routing Protocol uses History of Encounters and Transitivity [11]. Here user moves in predictable fashion based on history of encounters; from that delivery predictability is calculated. Delivery predictability has two properties; one is a history of encounter and the second is transitivity. History of an encounter is previously how many times any two user had contact. If a user has visited a location several times than probability to visit that location again is an increase. Transitivity property is based on the observation that if node A frequently come in contact with node B, and node B frequently encounters or come in contact with node $\mathrm{C}$, then node $\mathrm{C}$ is a better node to forward messages destined for node A [11]. Here node means the user.

ProPhet algorithm first calculates delivery predictability and based on that messageforwarding strategy will decide. Initially constant high delivery predictability is assigned to each node and based on nodes encounters matrix of delivery predictability is updated. If no encounters occur for a particular node than the delivery predictability of that user, reduce by aging constant [11]. Than transitivity, property is used to calculate delivery predictability of user. For forwarding in starting no path is available, so the message is simply buffered with that node than based on the delivery probability of that node to transfer messages to the destination is higher than threshold [11] then message is transferred to the destination.

The Prophet uses single copy of the message, so the overhead ratio of ProPhet is slightly less than Epidemic, but in ProPhet, node contains message until an encounter for the message occur, so network overhead is high until encounter for message will occur. Therefore, overhead of ProPhet is higher than other routing protocol like SimBet and Spray and Wait.

\section{4) SimBet}

SimBet combine to social characteristics Similarity and Betweenness Centrality. As social characteristics of a network are less volatile and long-term SimBet uses two characteristics among them is Similarity and Betweenness Centrality [8],[12]. Here Node Similarity is used to find whether nodes reside to same cluster and centrality is used to identify ties between two clusters.

SimBet algorithm used new Egocentric Betweenness metric based on ego network analysis to locally determine a node's centrality within the network [12]. In the SimBet algorithm, locally calculated centrality matrix used instead of globally calculated centrality matrix for the network. Globally calculated centrality matrix is complex and it requires all network information. 
Centrality means the head person in the network; local centrality matrices are calculated for each node, so full network information is not necessary. Betweenness Centrality is measure number of shortest path via certain node [8]. Here, node calculates Betweenness value locally based on equation [12] and within cluster node with higher Betweenness value is considered as the central node. The number of common nodes between individual within the cluster defines similarity, similarity shows the degree of separation [8].

From the value of Similarity and Betweenness, Similarity and Betweenness utility are calculated and then from that SimBet utility matrix is calculated for any node to deliver a message to the destination node. The SimBet utility value is between 0 and 1 [12]. For transmitting message to any destination node $\mathrm{d}$, Similarity utility and Betweenness utility of node $\mathrm{n}$ compare to node $\mathrm{m}$ is given below equation [12]:

$$
\begin{aligned}
\operatorname{SimUtil}_{n}(d) & =\frac{\operatorname{Sim}_{n}(d)}{\operatorname{Sim}_{n}(d)+\operatorname{Sim}_{m}(d)} \\
\operatorname{BetUtil}_{n}(d) & =\frac{\operatorname{Bet}_{n}(d)}{\operatorname{Bet}_{n}(d)+\operatorname{Bet}_{m}(d)}
\end{aligned}
$$

Where $\operatorname{Sim}_{n}$ Similarity of node $\mathbf{n}, \operatorname{Bet}_{n}$ is Ego Betweenness of node $\mathbf{n}, \operatorname{SimUtil}_{n}(d)$ is Similarity utility and $\operatorname{BetUtil}_{n}(d)$ is Betweenness utility of node $\mathbf{n}$ to transfer message to the destination $\mathrm{d}$, similar notations applicable for node $\mathrm{m}$ of utility is applicable for node $\mathrm{m}$.

The SimBet utility of node $\mathrm{n}$ for delivering message to node $\mathrm{d}$ is normalize weighted combination of Similarity utility and Betweenness utility [12]:

$$
\operatorname{SimBetUtil}_{n}(d)=\alpha \operatorname{SimUtil}_{n}(d)+\beta \operatorname{BetUtil}_{n}(d)
$$

Here, $\alpha+\beta=1, \alpha$ and $\beta$ are tunable parameters. In the simulation, we took both parameter as 0.5 to give equal importance to Similarity and Betweenness [8], [12]. SimBet forward only a single copy of a message, so the delivery cost and overhead of SimBet is less than Epidemic and Spray and Wait.

\subsection{Mobility Model}

DTN has an environment where the frequent partition in transmission path is there or we can say end-to-end connectivity is not normal. Messages are transmitted to the destination via intermediate nodes. To understand the mobility of user and gain realism, mobility models are developed using practical traces and by using synthetic hypothesis. Many traditional mobility models are still widely used to support easy DTN protocol evaluation. They usually cover only selected mobility characteristics, whereas synthetically generated node mobility models allow for fine-tuning in many aspects [13]. Here, human mobility model SLAW is discussed, which we are utilizing in simulation.

\section{1) SLAW}

SLAW is the human mobility based model, which has five statistical features to define human mobility model as listed above in section I.SLAW is composite model, and it produces synthetic mobility traces. Slaw also includes virtual features of mobility created by the user. Synthetic traces produced by SLAW give unique features in terms of performance evaluation of various routing protocols [13]. SLAW model capture heterogeneous mobility area of user with all TLW features, so it gives more realistic result than TLW, by this heterogeneous mobility people with common interest share similar area like people in the college campus, theater, city mall etc. SLAW model also use LATP [15] algorithm to find a user's next waypoint to visit. This is to get better performance of routing protocols over the random mobility models.

Five statistical feature of SLAW mobility model are discussed below:

- Truncated power-law flights and pause-times [14],[15]: 
Flight length and Pause time both follows Power- Law distributions. A flight length is defined to be a long straight-line trip from one location to another that a particle makes without a directional change or pause [14]. Pause time is duration when a user stays at one point before starting a new flight or we can say duration between two flights.

- Heterogeneously bounded mobility areas [15]: People mostly move only within their own confined areas of mobility, Different people may have widely different mobility areas.

- Inter-Contact Times (ICTs) [15]: The times elapsed between two successive contacts of the same person. ICTs can also be modeled by a truncated power law distribution.

- Fractal waypoints [15]: Derived From the analysis of the GPS traces of human walks Waypoints implies that people are always more attracted to more popular place.

- Least-Action Trip Planning (LATP) [15]: People are more likely to visit destinations nearer to their current waypoint.

Routing performance of SLAW effectively expresses mobility patterns arising from people with some common interests or within a single community like students in the same university campus or people in theme parks where people tend to share common gathering places [15].

\section{Proposed Routing Algorithm:Multi-Copy SimBet with Pause}

In proposed routing algorithm, with the Similarity utility and the Betweenness utility of SimBet, a new factor of utility is added as "Pause Time utility". This method uses "Pause Time" utility as the third parameter in the utility calculation of algorithm "Pause Time" of the nodes follows the Power Law distribution, which indicates if in the past a node take short pauses several times then probability of that node to take short pauses increases in future. By utilizing this phenomenon in this algorithm, we have calculated pause time from the past history of node pauses. By considering node, which has lower pause time as an active node for communication, the priority is given to the node, which has lower pause time as information carrier. To get delivery probability closed to Epidemic, make an original SimBet as multi-copy by spraying more than one copy in the network. "Multi-copy SimBet with Pause" routing algorithm message forwarding mechanism is similar to the original SimBet routing. In this algorithm, durations of node's pauses are extracted based on node mobility. The Pause Time Utility calculation for proposed method is given below:

$$
\operatorname{PUtil}_{n}(d)=\frac{P_{n}(d)}{P_{n}(d)+P_{m}(d)}
$$

Where $P_{n}$ Pause Time of node $\mathrm{n}, P_{m}$ is Pause Time of node $\mathrm{m}, \operatorname{PUtil}_{n}(d)$ is Pause Time utility of node $\mathrm{n}$ to transfer message to the destination d. Similar formula of utility is applicable for node $\mathrm{m}$.

The overall utility calculation of "Multi-copy SimBet with Pause" is a weighted combination of all three, Similarity utility, Betweenness utility and Pause Time utility. For overall utility of node, $n$ with respect to node $\mathrm{m}$ to transmit message to destination $\mathrm{d}$ is calculated from below equation:

$\operatorname{SimBetPUtil}_{n}(d)=\alpha \operatorname{SimUtil}_{n}(d)+\beta \operatorname{BetUtil}_{n}(d)+\gamma \operatorname{PUtil}_{n}(d)$

Where $\operatorname{SimBetPUtil}_{n}(d)$ is the overall utility of node $\mathrm{n}$ to transfer message to destination $\mathrm{d}$. Here, $\alpha+\beta+\gamma=1, \alpha, \beta$ and $\gamma$ are tunable parameters. In the simulation, we took all three parameter 0.33 to give equal importance to Similarity, Betweenness and Pause time. 


\section{Simulation}

Here simulation is preferred rather doing mathematical analysis.

\subsection{Simulation setup}

Opportunistic Networking Environment (ONE) simulator is used for simulation of routing protocol. Ten traces files are generated for particular numbers of user and to get accuracy in result Monte Carlo simulation method used for averaging of the results of ten traces file. In the simulation pauses of node calculated from first 10minutes of simulation.

Simulation parameters are listed in below in table:

\begin{tabular}{|c|c|}
\hline Parameters & Values \\
\hline Simulator & ONE \\
\hline Simulation Time & $1800 \mathrm{sec}$ \\
\hline Numbers of Nodes & 10 to 50 \\
\hline Mobility Model & SLAW \\
\hline Message Size & $500 \mathrm{kB}$ \\
\hline Buffer Size & $10 \mathrm{MB}$ \\
\hline Terrain & $100 \mathrm{~m} \mathrm{X} 100 \mathrm{~m}$ \\
\hline Transmission Range & $30 \mathrm{~m}$ \\
\hline Update Interval & $1 \mathrm{sec}$ \\
\hline
\end{tabular}

\subsection{Simulation Results}

We plot four different parameters vs. number of users; these four parameters are message delivery probability, average end-to-end delay, overhead ratio and average hop count. Following graphs from fig. 1 to fig. 4 are Simulation of different routing algorithm like Spray and Wait, Epidemic, ProPhet, SimBet, Multi-copy SimBet and 'Multi-copy SimBet with Pause' using SLAW mobility model. In 'simulation Multi-copy SimBet' and 'Multi-copy SimBet with Pause', replicates message 4 times.

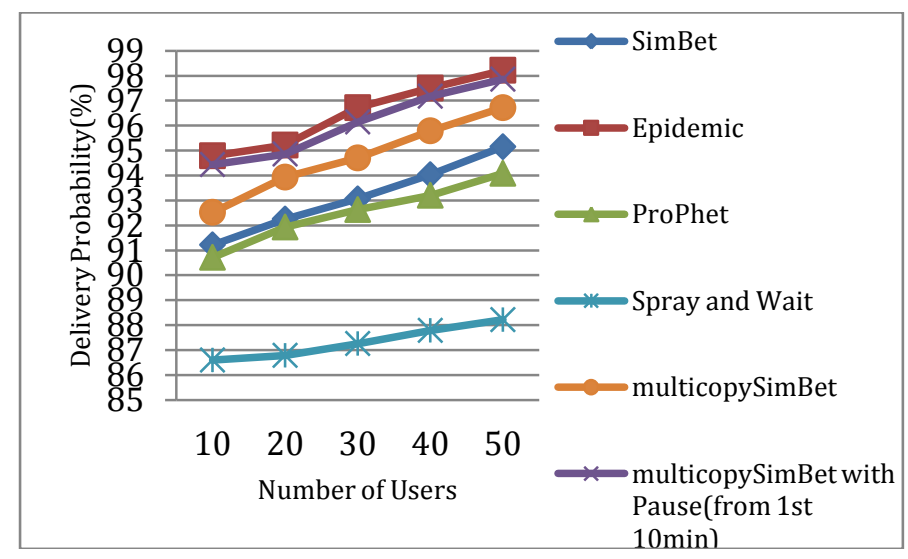

Figure 1: Delivery Probability VS Number of Users 
Figure 1 results show that the delivery probability of Epidemic Routing is highest among all Protocols because each and every host contains message copy, so message delivery probability is highest among all. Compare to any other algorithm 'Multi-copy SimBet with Pause' give results which maximum close to epidemic.

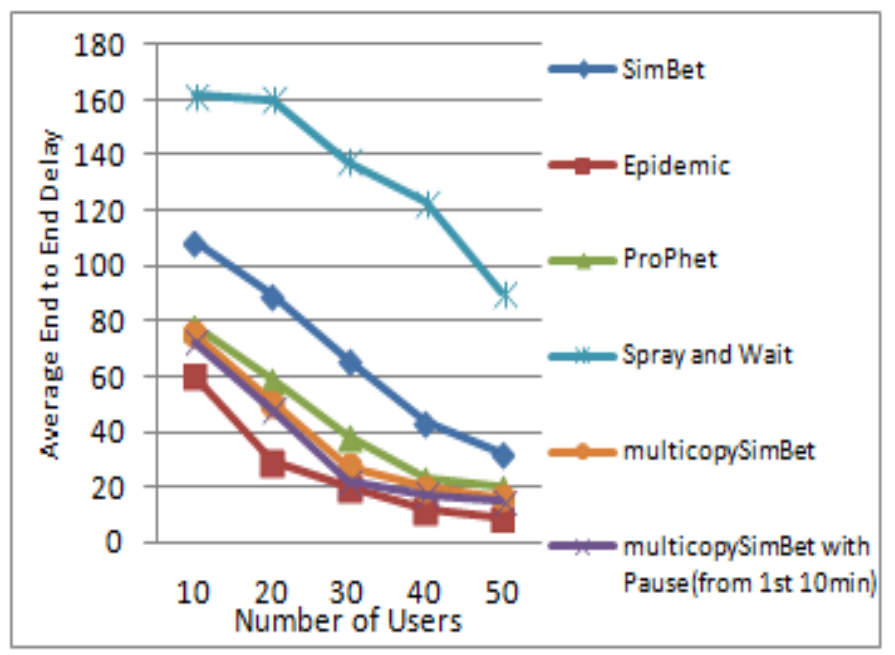

Figure 2: Average End to End Delay VS No. of Users

Figure 2 shows a higher end to end delay in Sray and Wait because it Wait until all messages are delivered, Epidemic has lower latency obviously because each and every host has message copy. SimBet slightly performs bad compare to ProPhet. Multi-copy SimBet and 'Multi-copy SimBet with Pause' perform close to epidemic which is better compared to original SimBet.

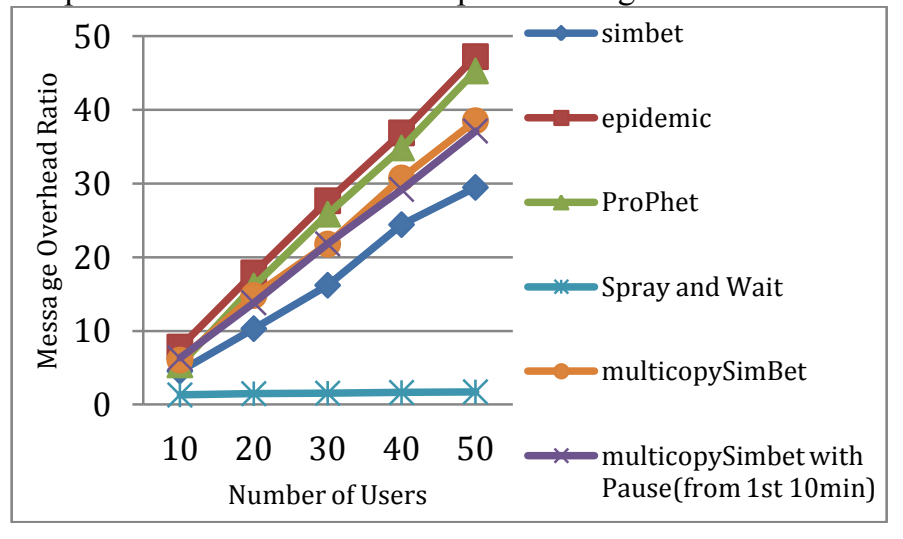

Figure 3: Overhead Ratio VS Number of Users

Figure 3 shows the highest overhead ratio of Epidemic because it has highest message copies. In ProPhet user hold message until an encounter for the messages occurs, so also it has heigher overhead. Multi-copy SimBet and 'Multi-copy Simbet with Pause' also have higher overhead because both follows replication of message concept to get higher message delivery. SimBet and Spray and Wait give good performance in terms of overohead. 


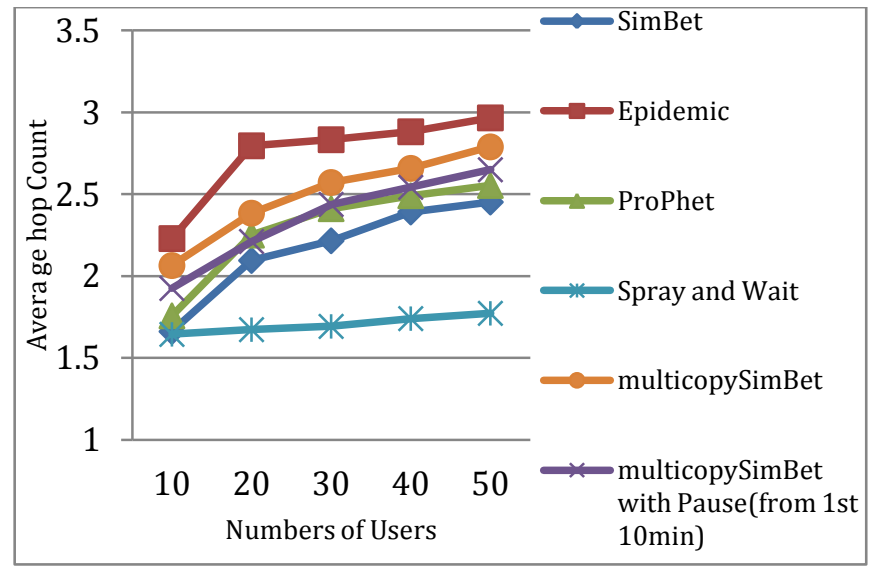

Figure 4: Average hop count VS No. of Users

Figure 4 shows highest average hop count of Epidemic because it has no scheme for effective forwarding, message travel hop to hop for delivery. Because of replication of the message in Multi-copy SimBet and 'Multi-copy SimBet with Pause', the hop count is increased than original SimBet. Spray and Wait have a lowest hop count because it spray only necessary copy of the message and wait until delivery of all messages.

Following figure 5 and figure 6 shows graphs for message delivery ratio by varying transmission range and Time to live (TTL). TTL is define for message life, how long message lives in network. TTL is defined in unit of minute.

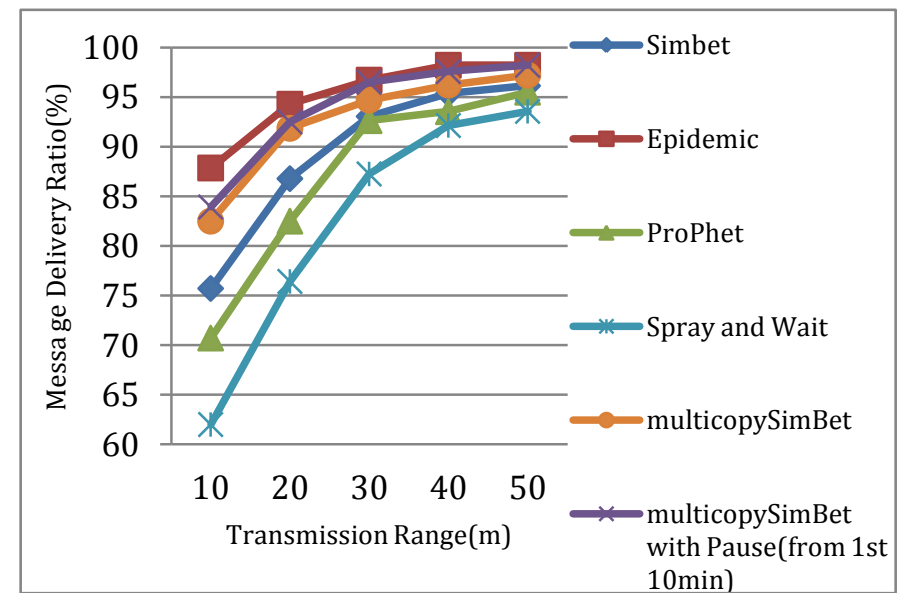

Figure 5: Message Delivery Probability VS Transmission Range

Figure 5 shows as transmission range increases node coverage area increase and more nodes come in contact, so all routing algorithm's message delivery probability increases. 'Multi-copy SimBet with Pause' goes closer to epidemic as transmission range increases. 


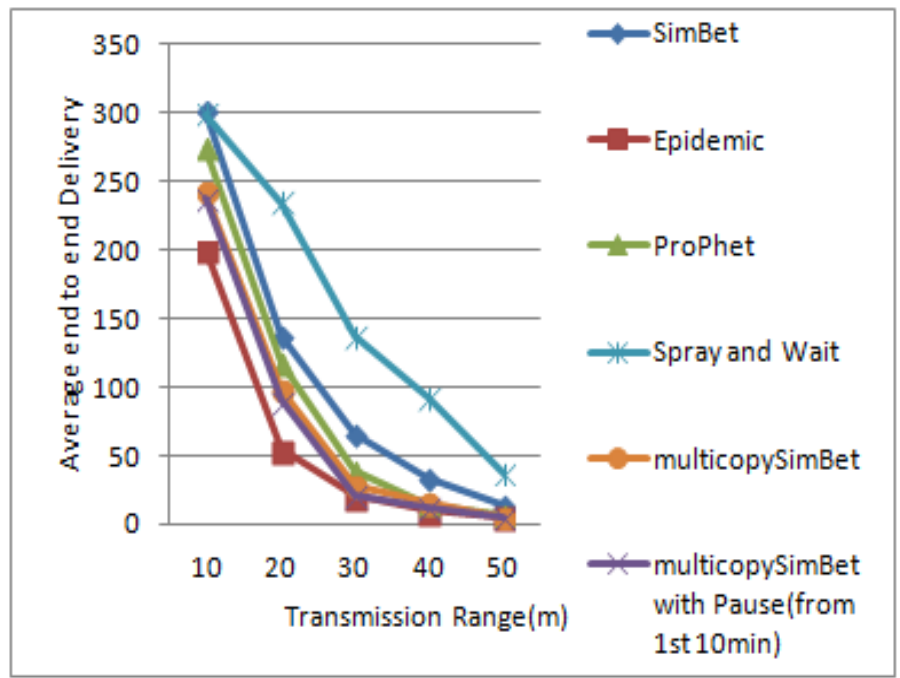

Figure 6: Average End-to-End Delay VS Transmission Range

Figure 6 shows that as transmission range increases node coverage area increases, so average delay of node is reduce, "multicopySimBet with Pause", have good performance compare to SimBet, Spray and Wait and ProPhet. Epidemic has least delay because node transmits message copy to each node come in contact.

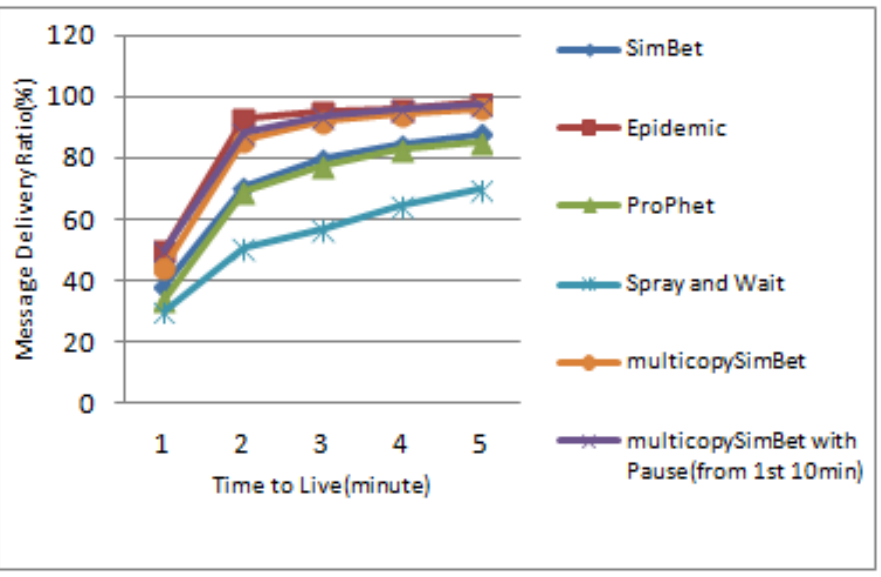

Figure 7: Delivery Probability VS Time to live

Figure 7 shows that long life of node is responsible for higher message delivery. Here "multicopySimBet with Pause" give delivery probability close to Epidemic for lower values of Ttl also compare to other all existing routing algorithms. 


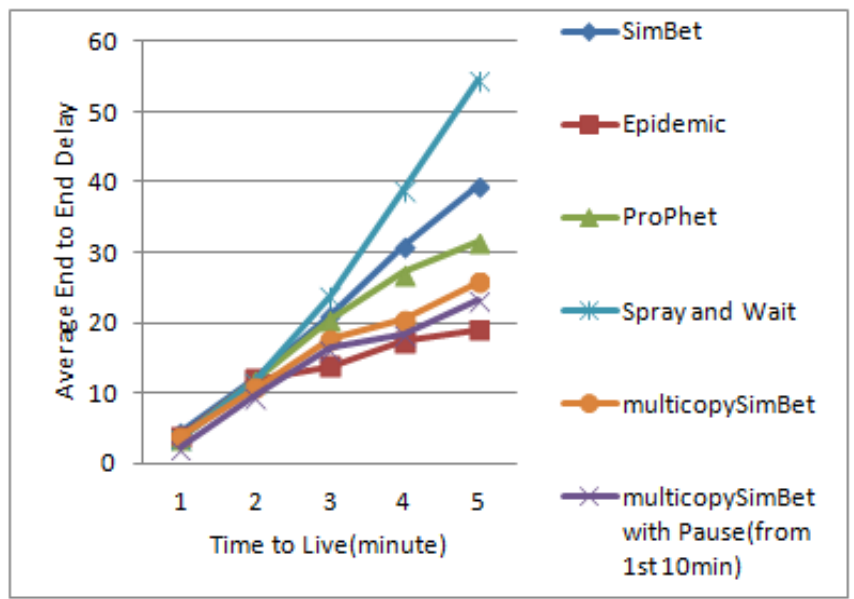

Figure 8: Average End-end Delay VS Time to live

Figure 8 shows that average end-to-end delay of all algorithms increases as message life in network increases. Here also proposed algorithm give result close to epidemic as compare to others.

\section{Conclusions}

Performance of 'Multi-copy SimBet with Pause' gives better result compared to Multi-copy SimBet and other existing algorithm, so we can justify DTN using the past history of the pauses of the human based mobility shows much better improvement in performance. Among all these algorithms 'Multi-copy SimBet with Pause' is good choice with considerably close deliver probability with Epidemic. In addition, it has lower overhead ratio and lower end-to-end delay

\section{Acknowledgement}

This research work is a part of GUJCOST MRP on "Air Pollution Monitoring system for Anand City based on Delay Tolerant Communication technology of Cell Phones". We would like to express our gratitude and sincere thanks to GUJCOST.

\section{References}

[1] Kevin Fall, "A Delay-Tolerant Network Architecture for Challenged Internets", SIGCOMM, August 25-29, 2003.

[2] Hervé Ntareme, Marco Zennaro, Björn Pehrson, "Delay Tolerant Network on smartphones: Applications for communication challenged areas", ACM, New York, USA, 26 sept. 2011.

[3] emios G. Voyiatzis, "A Survey of Delay- and Disruption-Tolerant Networking Applications", JOURNAL OF INTERNET ENGINEERING, VOL. 5, NO. 1, JUNE 2012, 331-344. 
[4] Shally, Harminder Singh Bindra, Mamta Garg, "Performance Evaluation of Rapid and SprayAnd-Wait Dtn Routing Protocols Under Black Hole Attack" IJRET, Volume: 03 Issue-01, Jan-2014.

[5] Sushant Jain, Kevin Fall, and Rabin Patra, "Routing in a Delay Tolerant Network", In SIGCOMM, September 2004, 145-157.

[6] Evan P.C. Jones, Lily Li, Jakub K. Schmidtke, Paul A.S. Ward, "Practical Routing in DelayTolerant Networks", IEEE TRANSACTIONS ON MOBILE COMPUTING, VOL. 6, NO. 8, AUGUST 2007, 943-959.

[7] Evan P.C. Jones and Paul A. S. Ward, "Routing Strategies for Delay Tolerant Networks," Journal of Computer Communication Journal, 2008.

[8] Ying Zhu, Bin Xu, "Survey of Social-Based Routing in Delay Tolerant Networks: Positive and Negative Social Effects" IEEE COMMUNICATIONS SURVEYS \& TUTORIALS, VOL. 15, NO. 1, FIRST QUARTER 2013, 387-401.

[9] Amin Vahdat and David Becker. Epidemic routing for partially-connected ad hoc networks. Technical report, 2000.

[10] Thrasyvoulos Spyropoulos, Konstantinos Psounis, and Cauligi S.Raghavendra. Spray and wait: An efficient routing scheme for intermittently connected mobile networks, 2005.

[11] A. Lindgren, A. Doria, and O. Schel'en, "Probabilistic routing in intermittently connected networks," SIGMOBILE Mob. Comput. Commun.Rev., vol. 7, no. 3, pp. 19-20, 2003.

[12] E. M. Daly and M. Haahr, "Social network analysis for routing in disconnected delay-tolerant manets," in MobiHoc '07 Proc. 8th ACM international symposium on Mobile ad hoc networking and computing,2007, 32-40.

[13] M Shahzamal, M F Pervez, M A U Zaman and M D Hossain,"MOBILITY MODELS FOR DELAY TOLERANTNETWORK: A SURVEY", IJWMN, Vol. 6, No. 4, August 2014

[14] Injong Rhee, Minsu Shin, "On the Levy-walk Nature of Human Mobility" IEEE INFOCOM 2008, 1597-1605.

[15] Kyunghan Lee, Seongik Hong, "SLAW: Self-Similar Least-Action Human Walk" IEEE, VOL. 20, NO. 2, APRIL 2012, 515-529. 